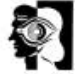

columns
Oluwatayo \& Friedman that there is need for a national approach and guidance on minimum standards.

The Mental Health (Care and Treatment) (Scotland) Act 2003 enshrines in law a requirement for National Health Service (NHS) boards in Scotland to make appropriate provision for admitting mothers with their babies for treatment of mental illness in the postnatal period. The Act also encourages NHS boards to collaborate in delivering services. Recent guidance (Scottish Executive Health Department, 2004) emphasises the need to develop community, maternity liaison and specific primary care services in tandem with in-patient provision, and sets minimum standards for care for both mother and baby. A formal Scottish Executive Health Department review of progress towards implementation of the Act in October 2005 is ongoing. Inevitably this has led to an approach that is national in aspiration.

Scottish provision remains patchy, with one six-bed unit serving the west, but plans are rapidly developing in other areas through regional planning structures, with close communication between those involved in running existing services and those commissioning new provision. What has become clear from our experience is that specialist provision must involve collaboration across wide geographical/ population areas to ensure viability of services and development of appropriate knowledge and expertise.

CONFIDENTIAL ENQUIRY INTO MATERNAL AND CHILD HEALTH (2004) Why Mothers Die 2000 -2002 Report on Confidential Enquiries into Maternal Deaths in the United Kingdom. London: Royal College of Obstetricians and Gynaecologists.

SCOTTISH EXECUTIVE HEALTH DEPARTMENT (2004) A Framework for Mental Health Services in Scotland: Perinatal Mental IIIness/Postnatal Depression Admission and Support Services. Edinburgh: Scottish Executive Health Department.

${ }^{*}$ Roch Cantwell Consultant in Perinatal Psychiatry, Glasgow Perinatal Mental Health Service, Department of Psychiatry, Southern General Hospital Glasgow G51 4TF, e-mail: Roch.Cantwell@glacomen. scot.nhs.uk, Karen Robertson Nurse Consultant, Glasgow Perinatal Mental Health Service, Chair Scottish Executive Health Department Working Group on Perinatal Mental Illness Services

\section{Transfer from child to adult mental health services}

Singh et al (Psychiatric Bulletin, August 2005, 29, 292-294) discuss the risk of disrupted care for young people who outgrow child and adolescent menta health services (CAMHS)

Lincolnshire Partnership NHS Trust has a protocol for good practice surrounding transfer of a young person's care from child to adult mental health services. However, in an audit of these procedures involving 82 young people aged 17 or 18 years who were discharged from three of our community CAMHS teams over a 2-year period, only seven were transferred to adult services. CAMHS clinicians identified 32 other young people who left the service with unresolved mental health problems: a suitable adult service could not be found for one young person, 21 young people dropped out of CAMHS and ten young people did not want to be referred to adult services.

The paucity of psychological therapies in adult mental health services created difficulties for CAMHS clinicians in finding suitable follow-on services. Perhaps the prospect of an inevitable ending with no further support contributed to the high drop-out rate of young people approaching the end of the service available to them in CAMHS? Some young people clearly said they did not want to have to 'start from the beginning' in establishing a therapeutic relationship with a new worker. Others were perhaps influenced in declining ongoing care by the perceived stigma of adult services.

Our audit findings add to the evidence that the current differing perspectives of CAMHS and adult mental health services create gaps in services through which vulnerable young people fall.

AnneThompson Consultant in Child and Adolescent Psychiatry, Lincolnshire Partnership NHS Trust, Lincoln LN2 5RT, e-mail: anne.thompson@|pt. nhs.uk

\section{Are psychiatrists real doctors?}

The survey of psychiatric trainees in Scotland by Dr Robinson (Psychiatric Bulletin February 2005, 29, 62-64) showed that a significant amount of physical healthcare is being provided by psychiatric trainees.

In my experience, south of the border the situation is no different, particularly in psychiatric long-stay facilities such as rehabilitation units and forensic units where a large degree of physical morbidity exists. Cormac et al (2004) reported high rates of avoidable health risks such as smoking, obesity, central weight distribution and excessive weight gain.

The role of the trainee is to identify and manage problems for which they often may have received no formal training. After completion of pre-registration house jobs, direct entry to psychiatric training schemes is not uncommon. The notion of managing, for example, an individual's diabetes, hypertension or obesity may be quite alien never mind being able to recognise strange skin complaints and other problems commonly encountered in primary care.

I have experience and training in primary care which I have found invaluable in dealing with my patients' physical health problems. The National Service Framework for Mental Health requires health promotion and appropriate access to and delivery of primary care for patients with mental disorders (Department of Health, 1999). It may be of value to consider the training needs of psychiatric trainees with regards to management of physical health problems.

CORMAC, FERRITER, M BENNING, R et al(2005) Physical health and health risk factors in a population of long-stay psychiatric patients. Psychiatric Bulletin, $29,18-20$

DEPARTMENT OF HEALTH (1999) National Service Framework for Mental Health: Modern Standards and Service Models. London: Department of Health

Harpreet Pannu Staff Grade Psychiatrist, Rampton Hospital, Retford DN22 OPD, e-mail: Harpreet.pannu@nottshc.nhs.uk

\section{Physical health of patients in rehabilitation and recovery}

I read with interest the article by $D r$ Greening (Psychiatric Bulletin, June 2005, 29, 210-212). I have recently undertaken an audit of the physical healthcare of patients in our rehabilitation and recovery unit in Warwick. Unfortunately, my preliminary results show a similar picture to that reported by Dr Greening.

However, we do have a local general practitioner (GP) who has two sessions allocated per week for the review of any physical health problems: the type of 'shared care approach' suggested by Lester (2005) and Bickle (2005). It must be stressed though that it is not the responsibility of our GP colleagues to trawl through reams of notes (which most rehabilitation patients have) but rather up to the psychiatric team to ensure that patients are having appropriate investigations that can then be discussed with primary care.

For my audit I initially drew up a 'checklist' (standards) of the investigations that patients should have depending on what type of medication they are prescribed and how often, if at all, this ought to be repeated. I used the Maudsley Prescribing Guidelines, British National Formulary and consulted pharmaceutical companies in drawing up the standards for each psychotropic agent one must not forget mood stabilisers and antidepressants that also require monitoring. Although rather time-consuming, it is a more rigorous method than collating the views of colleagues as done by Pitman (2005) prior to audit and is better than a battery of 'routine tests' which may be incomplete.

In addition, we have put together a health screen protocol for each patient that not only looks at issues such as diet, 